\title{
Trigeminal Nerve
}

National Cancer Institute

\section{Source}

National Cancer Institute. Trigeminal Nerve. NCI Thesaurus. Code C12806.

The fifth set of paired nerves of the face that emerge from the brain steam. These

nerves have sensory and motor functions in the face, oral cavity, and nasal cavity. 